Article

\title{
Optical Properties of Titanium in the Regime of the Limited Light Penetration
}

\author{
Lukasz Skowronski (D) \\ Institute of Mathematics and Physics, UTP University of Science and Technology, Kaliskiego 7, \\ 85-796 Bydgoszcz, Poland; lukasz.skowronski@utp.edu.pl
}

Received: 4 February 2020; Accepted: 18 February 2020; Published: 20 February 2020

\begin{abstract}
In this study, the titanium layers from 12 to $1470 \mathrm{~nm}$ thick were fabricated by using the method involving dynamically changed working gas pressure (gas injection magnetron sputtering). The influence of the deposition time on the optical and electrical properties of $\mathrm{Ti}$ films, as well as on their microstructure, are considered. The samples are investigated by means of spectroscopic ellipsometry, atomic force microscopy, X-ray diffraction, and confocal optical microscopy. Additionally, for the Ti layers, the sheet resistance was determined. The produced coatings exhibit privileged direction of growth (002). The obtained results show a gradual increase in the mean relaxation time of free-carriers with the increase in the thickness of titanium film. However, the plasma energy exhibits maximum for the coating with the thickness of $93 \mathrm{~nm}$. For such thickness, the lowest value of optical resistivity (about $200 \mu \Omega \mathrm{cm}$ ) was observed. It was found that the dc- and optical resistivity exhibit similar values for titanium films with thickness up to $93 \mathrm{~nm}$. For thicker Ti layers, significant differences in resistivities (dc- and optical) were noticed. The behavior of the Drude parameter (the plasma energy), calculated optical resistivity, and discrepancies between values of optical and dc-resistivities for thicker Ti coatings can be explained as a result of the limited light penetration.
\end{abstract}

Keywords: magentron sputtering; titanium; optical properties; resistivity; penetration depth

\section{Introduction}

The microstructural and optical properties of the materials synthesized in the vacuum chamber strongly depend on the growing conditions (e.g., a pressure of working/reactive gas, deposition rate, substrate temperature, and the bias applied) [1]. There are various methods used to increase energy of condensed particles, e.g., heating of the substrate (and/or post-deposition annealing) [2,3], plasma bombardment [4], increasing of the power of the supplier [3,5]. Sometimes the additional voltage, the bias, is applied during the coating formation process $[4,6,7]$. Those methods lead to increasing the kinetic energy of particles dissipated due to the inter-particle collisions. The above mentioned problem is very important since the microstructure and phase composition of nitrides/oxides strongly depend on the energy of condensed particles and the effect of macroscopic properties of coatings; hardness, corrosion resistance, and optical properties. Moreover, as shown in Reference [8], the energy conditions during the layer formation are associated only with condensable particles if the temperature of substrate is lower than $180{ }^{\circ} \mathrm{C}$.

A completely different approach is applied in gas injection magnetron sputtering (GIMS) technique [9-11]; the variable pressure of plasma forming gas is used to control the plasma process. The impulse injection of a working gas into the vacuum chamber elongates the mean free path of gas phase particles and decreases inter-particles' collisions probability. This means that the kinetic energy of gas phase particles is practically not dissipated before their condensation on the substrate. The GIMS method was successfully used to produce decorative multilayers [12-16]. The recent investigation 
clearly shows that applying the GIMS technique allows for synthesizing the nanocrystalline rutile phase of $\mathrm{TiO}_{2}$ directly during the deposition on the unheated substrate [17].

The aim of this work is to examine the influence of the thickness of titanium films (from $12 \mathrm{~nm}$ to $1470 \mathrm{~nm}$ ) formed using the GIMS method on their microstructure, optical and electrical properties. It should be noted that microstructural properties of Ti coatings prepared under different conditions and using various modifications of magnetron sputtering methods are described in literature [2-7,18-21]; however, their optical constants and/or opto-electronic properties are described only in a few publications [20,22-24]. Additionally, the results related to the optical constants of Ti can b found in Reference [12,14,15]. It should be noted that the optical properties (the complex dielectric function, reflectance and transmittance spectra) of semitransparent titanium coatings (the thickness of a layer below $50 \mathrm{~nm}$ ) were described in Reference $[13,14]$. However the description was limited and related to the influence of the complex dielectric function of Ti deposited on glass on reflectance and/or transmittance spectra of $\mathrm{Ti} /$ glass $\mathrm{Ti} / \mathrm{Al}$ systems and then on the color formation (and transparency) of $\mathrm{TiO}_{2} / \mathrm{Ti} /$ glass and $\mathrm{TiO}_{2} / \mathrm{Ti} / \mathrm{Al}$ structures. Some of the results included in Reference [13] are presented in this publication as a part of a larger research, related to the systematic investigation on influence of the thickness (two orders of magnitude) of titanium coatings on their properties, and are precisely marked in the presented manuscript.

In general, the resistivity of metallic layer deposited at established growth conditions decreases with the increase in the thickness. In the presented manuscript it was shown that the behavior of the optical resistivity may be reverse when the metallic coating is thick enough, while the dc-resistivity keeps the above-mentioned trend. This effect has been explained as a result of the limited penetration of light (the optical response is related only to a top part of the Ti coating).

\section{Materials and Methods}

\subsection{Sample Preparation}

The Ti layers were deposited on Bk7 glass plates (1 mm thick) using the GIMS method applying the industrial magnetron line in the Bohamet company [25]. Before the coating production the substrate was cleaned in a solution based on a mixture of alcohols and surfactants. Moreover, the plasma cleaning of the glass was performed before the deposition of titanium coatings.

To deposit titanium coatings, two linear Ti targets (grade 1), $2500 \times 100 \times 10 \mathrm{~mm}$, were used. The target-substrate distance was $140 \mathrm{~mm}$. The DPS-type power supplier (Dora Power Systems) [26] was used during the deposition process. The power supply was operated in $100 \mathrm{kHz}$ sinusoidal pulses grouped in the $2 \mathrm{kHz}$ unipolar pulse packets. The detailed description of the GIMS method therein voltage characteristics can be found in Reference [9,17,27-29].

The pressure in the vacuum chamber before the deposition was $p_{0}<0.01 \mathrm{~Pa}$, while, during the deposition, the average pressure was $p=0.06 \mathrm{~Pa}$. The sputter gas (argon; $5 \mathrm{~N}$ ) was injected as $3 \mathrm{~Hz}$ impulses $[9,17]$. The Ti layers with the various thickness were produced by changing the deposition time from $11 \mathrm{~s}$ to $2240 \mathrm{~s}$.

\subsection{Sample Characterization}

The Atomic Force Microscope (AFM) Innova (Bruker) device (with the standard Si tips for a tapping mode) was used to examine the surface topography of the Ti films. The scan size was $2 \mu \mathrm{m} \times 2 \mu \mathrm{m}$. The roughness parameter $R_{a}$ (the arithmetical mean deviation of the assessed profile) was determined using the NanoScope Analysis software (version 1.40). The $R_{a}$ quantity is defined as:

$$
R_{a}=\frac{1}{N} \sum_{j=1}^{N}\left|Z_{j}\right|
$$


In Equation (1), $Z_{j}$ is the current surface height value, while $N$ is the number of points measured.

The XRD measurements were performed using PANanalytical X-Pert PRO X-Ray diffractometer with Ni-filtered $\mathrm{Cu} \mathrm{K} \alpha$ radiation $(\lambda=1.5418 \AA)$.

A sheet resistance (4PP) was measured with a four-point probe (from Jandel Engineering Ltd.) with the Keithley nanovoltmeter. The measurements were performed at several points on the surface of a titanium film.

Ellipsometric azimuths $\Psi$ and $\Delta$ were measured for two or three angles of incidence $\left(65^{\circ}\right.$ and $75^{\circ}$ for non-transparent or $65^{\circ}, 70^{\circ}$, and $75^{\circ}$ for semitransparent samples) in the NIR-vis-UV spectral range (193-2000 nm; 0.6-6.5 eV) by the V-VASE (from J.A.Woollam Co., Inc.) device. Additionally, for semitransparent samples the transmittance measurements for near-normal incidence were performed by means of the Cary 5000 spectrophotometer. The WVASE32 software (J. A. Woollam Co., Inc.) was used to perform the fit procedure. The spectroscopic ellipsometry (SE) technique, combined with transmittance measurements, was used to determine thicknesses of the rough layer and the titanium film (only semitransparent samples), as well as optical constants of the metallic coatings.

The thickness of the opaque titanium films was estimated from a linear interface layer/substrate profile using the confocal optical microscope (COM) Lext OLS 4000 (from Olympus). Those measurements were performed at ten points on the profile.

\section{Results and Discussion}

The thickness of titanium coatings $\left(d_{T i}\right)$ was established using the spectroscopic ellipsometry technique and the confocal optical microscope. Detailed description related to the determining of the thickness of Ti will be presented in the 'Optical and Electrical Properties of the Titanium Coatings' section; however, in the 'Results and Discussion' section, these thicknesses will be used as a part of the name of a sample (in the following form: $\mathrm{Ti}\left(d_{T i}\right.$ rounded to $\left.\left.1 \mathrm{~nm}\right)\right)$ and during discussion of the obtained results. Due to a strong dependence of microstructural, electrical and optical properties on the metallic layer thickness [30], the obtained results are discussed as a function of $d_{T i}$.

\subsection{Topography of Ti Coatings}

Figure 1 presents the surface topography of titanium coatings for different thickness. The AFM images for the thinner Ti films were presented in Reference [13]. One can see that these topographies show significant dependence on the time of deposition $\left(t_{T i}\right)$ and thereby on the thickness of the Ti film, especially for thicknesses larger than $50 \mathrm{~nm}$. The lateral grain size increases with $d_{T i}$ from about 50-100 nm for the Ti(12) sample to 100-300 nm for the Ti(1470) specimen. The values of $R_{a}$ for the produced metallic films (see Table 1) are in the range from about $2 \mathrm{~nm}$ (for Ti(12)) to about $8 \mathrm{~nm}$ (for $\mathrm{Ti}(1470)$ ). For the thicker titanium coatings the values of $R_{a}$ are negligibly low in comparison with the coating thickness. This result indicate that the obtained coatings are relatively smooth.
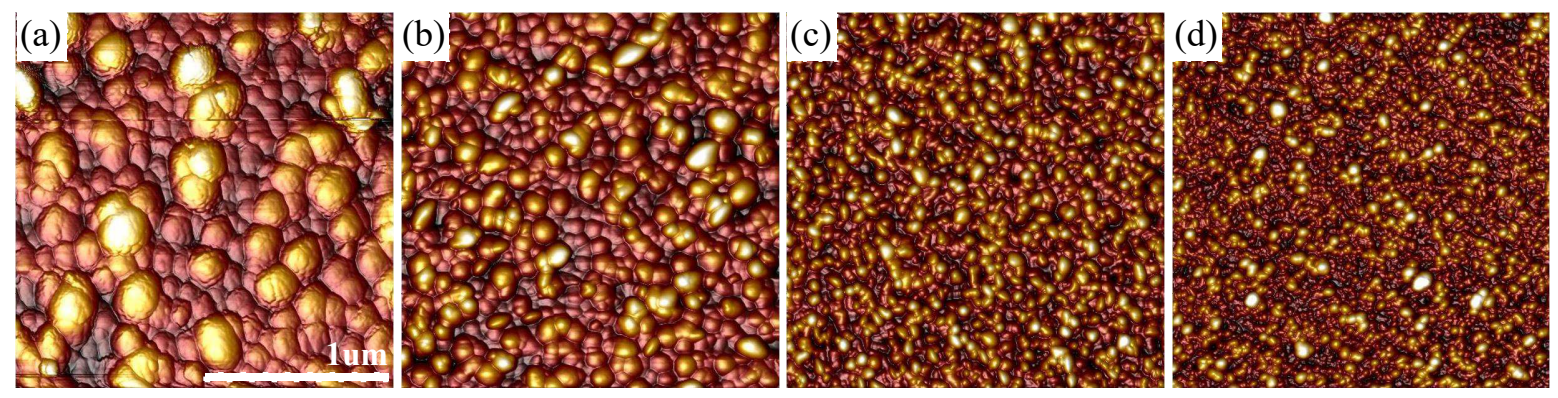

Figure 1. Atomic Force Microscope (AFM) images $(2 \mu \mathrm{m} \times 2 \mu \mathrm{m})$ of Ti layer surfaces for: (a) Ti(1470), (b) $\operatorname{Ti}(743),(\mathbf{c}) \operatorname{Ti}(363)$, and (d) $\mathrm{Ti}(93)$ samples. 
Table 1. Drude parameters ( $\hbar \omega_{p}$ and $\left.\hbar \Gamma\right)$, the mean relaxation time of conduction electrons $(\tau)$, the dc-resistivity $\left(\rho_{d c}\right)$, the optical resistivity $\left(\rho_{o}\right)$, and the sheet resistance $(R)$ of the Ti layers.

\begin{tabular}{ccccccc}
\hline Sample & $\hbar \omega_{p}(\mathbf{e V})$ & $\hbar \Gamma(\mathbf{e V})$ & $\tau(\mathbf{f s})$ & $\rho_{o}(\mu \Omega \mathbf{c m})$ & $\rho_{d c}(\mu \Omega \mathbf{c m})$ & $R(\Omega)$ \\
\hline $\operatorname{Ti}(12) *$ & $6.4 \pm 0.1$ & $2.40 \pm 0.16$ & $0.27 \pm 0.02$ & $422 \pm 25$ & $529 \pm 6$ & $434 \pm 7$ \\
$\operatorname{Ti}(16)^{*}$ & $6.7 \pm 0.1$ & $2.38 \pm 0.12$ & $0.28 \pm 0.02$ & $396 \pm 20$ & $465 \pm 5$ & $300 \pm 5$ \\
$\operatorname{Ti}(21)^{*}$ & $7.0 \pm 0.1$ & $2.26 \pm 0.14$ & $0.29 \pm 0.02$ & $338 \pm 21$ & $393 \pm 4$ & $189 \pm 3$ \\
$\operatorname{Ti}(26)^{*}$ & $7.8 \pm 0.2$ & $2.19 \pm 0.11$ & $0.30 \pm 0.02$ & $265 \pm 13$ & $299 \pm 3$ & $114 \pm 2$ \\
$\operatorname{Ti}(37) *$ & $7.9 \pm 0.2$ & $2.15 \pm 0.11$ & $0.31 \pm 0.02$ & $257 \pm 16$ & $281 \pm 1$ & $72.8 \pm 0.6$ \\
$\operatorname{Ti}(49)^{*}$ & $8.0 \pm 0.1$ & $2.11 \pm 0.13$ & $0.31 \pm 0.02$ & $221 \pm 16$ & $267 \pm 1$ & $54.7 \pm 0.5$ \\
$\operatorname{Ti}(93)$ & $8.7 \pm 0.2$ & $2.05 \pm 0.11$ & $0.32 \pm 0.02$ & $202 \pm 15$ & $193 \pm 1$ & $46.0 \pm 0.1$ \\
$\operatorname{Ti}(164)$ & $7.2 \pm 0.1$ & $1.58 \pm 0.05$ & $0.42 \pm 0.01$ & $227 \pm 10$ & $185 \pm 1$ & $19.8 \pm 0.2$ \\
$\operatorname{Ti}(363)$ & $6.6 \pm 0.1$ & $1.31 \pm 0.06$ & $0.50 \pm 0.02$ & $220 \pm 10$ & $180 \pm 1$ & $10.9 \pm 0.2$ \\
$\operatorname{Ti}(743)$ & $6.3 \pm 0.1$ & $1.23 \pm 0.04$ & $0.53 \pm 0.02$ & $233 \pm 12$ & $173 \pm 1$ & $4.76 \pm 0.06$ \\
$\operatorname{Ti}(1470)$ & $5.7 \pm 0.1$ & $1.10 \pm 0.04$ & $0.60 \pm 0.02$ & $250 \pm 13$ & $162 \pm 1$ & $2.18 \pm 0.04$ \\
\hline
\end{tabular}

* values of $\hbar \omega_{p}, \hbar \Gamma$ and $\tau$ estimated for the examined Ti coatings are taken from Reference [13]; the values of $\rho_{d c}$ and $R$ for these coatings were not published earlier.

\subsection{XRD Measurements}

The XRD pattens recorded for the produced Ti coatings are presented in Figure 2. According to the \#ICSD 7-6265 card, $\alpha$-Ti (the hexagonal-close-packed form (hcp); latice constants: $a=2.950 \AA$, $c=4.685 \AA$ ) has three main diffraction peaks in the $2 \theta$ range from $35^{\circ}$ to $41^{\circ}$ (calculated for $\mathrm{Cu} \mathrm{K}_{\alpha}$ radiation) and three, less intensive, for $50^{\circ}<2 \theta<70^{\circ}$. The primary diffraction peaks at $2 \theta=35.1^{\circ}$, $38.2^{\circ}$ and $40.2^{\circ}$ correspond to reflections from (100), (002) and (101) planes, respectively. XRD patterns presented in Figure 2 exhibit only one peak, which could be attributed to reflections from planes with Miller indices (002). No signals at $2 \theta=35.1^{\circ}$ and $40.2^{\circ}$ were detected. A week signal recorded at $2 \theta$ $=34.5^{\circ}$ is associated with unfiltered $\mathrm{Cu} \mathrm{K} \mathrm{K}_{\beta}$ radiation and is visible in XRD patterns for $\operatorname{Ti}(743)$ and $\mathrm{Ti}(1470)$ samples due to a high intensity of the (002) peak. This indicates the strong texturization of coatings produced applying the GIMS method. Similar results, with only one peak from (002) planes, were previously obtained by Jung [6] for the Ti layers produced using grid-attached magnetron and relatively high value of the bias polarization $(-150 \mathrm{~V})$, as well as by Chawla [3] for the Ti coatings formed using a relatively high power density. Despite the fact that (002) orientation is preferred for titanium layers deposited on glass [20], the results indicate that only one growth direction is characteristic for high-energy condensed particles.

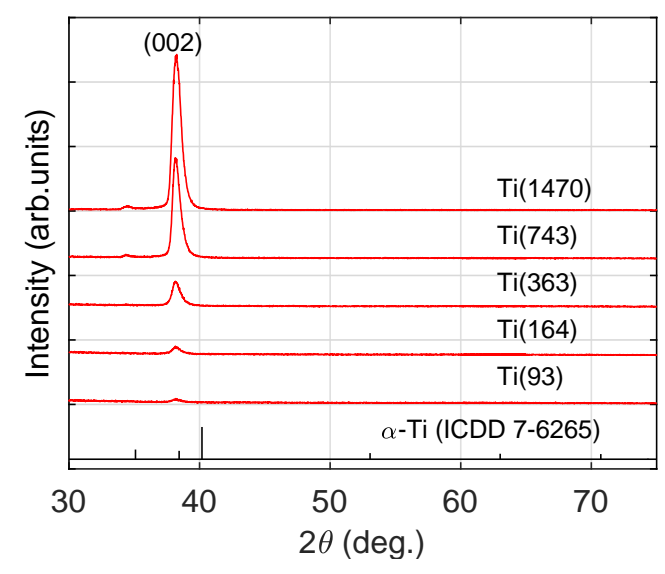

Figure 2. Diffractograms of the Ti layers.

The average size of nanocrystallites was estimated using the Scherrer formula:

$$
<D>=\frac{0.9 \lambda}{\beta \cos (2 \theta)} \text {. }
$$


In Equation (2), $\lambda$ is the $X$-ray wavelength $(\lambda=1.5418 \AA)$, and $\beta$ is the full-width at half-maximum (FWHM) of the Bragg diffraction peak at angle 20. The estimated grain size $\langle D\rangle$ is $7-10 \mathrm{~nm}$ with uncertainty at the level of 3-5 nm (see Table 1 ) and does not show the thickness of coating dependence.

\subsection{Optical and Electrical Properties of the Titanium Films}

Figure 3a shows a structure of titanium coating deposited on the glass substrate. To determine the complex dielectric function of titanium coatings, as well as the thickness of the Ti films, the five-phase (or three-phase-for thicker layers) optical model of a sample was used: ambient/rough layer/Ti layer/intermix layer/Bk7 glass (see Figure 3a). For thicker layers (samples with $d_{T i} \geq 93 \mathrm{~nm}$ ), the glass substrate and the intermix layer were omitted during the analysis of spectroscopic ellipsometry results.

(a)
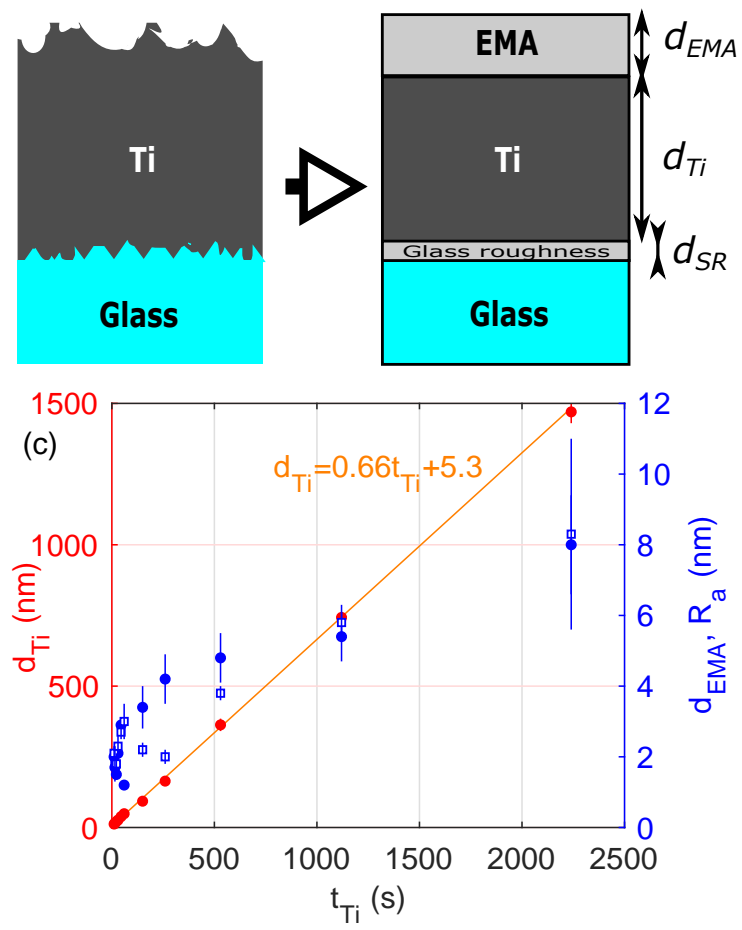

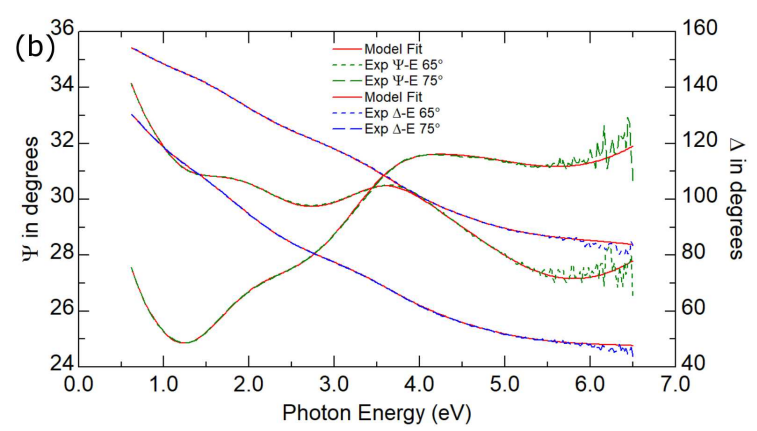

Figure 3. (a) A structure of the Ti coating deposited on the glass substrate and an optical model of a sample ( $d_{E M A}$ - the thickness of the rough layer, $d_{T i}$ - the thickness of the titanium layer). (b) Experimental $\Psi$ and $\Delta$ azimuths for two angles of incidence $\left(65^{\circ}\right.$ and $\left.75^{\circ}\right)$ and their model fits for the Ti(363) sample. The fit was performed using $P=12$ parameters $\left(\chi^{2}=1.6\right)$. (c) Thicknesses of the titanium coating $\left(d_{T i}\right)$ and the rough layer $\left(d_{E M A}\right.$; filled circles), as well as value of the $R_{a}$ parameter (open squares). The solid line represents the linear fit of $d_{T i}$ versus $t_{T i}$ dependence. EMA $=$ Effective Medium Approximation.

To describe optical constants of the rough layer, the two $\left(d_{T i} \leq 49 \mathrm{~nm}\right)$ or three (for $d_{T i} \geq 93 \mathrm{~nm}$ ) component Bruggeman Effective Medium Approximation (EMA) [31,32] model was used as a combination of optical properties of native titanium dioxide and titanium or void, native titanium dioxide and titanium (the assumed volume fraction of each medium was $1 / 2$ - for the two component structure or $1 / 3$ for the three component system). This approach to specification of the structure and optical properties of the rough layer for various thicknesses of titanium coatings was made for a more detailed description of the produced coatings. The optical constants of native $\mathrm{TiO}_{2}$ were taken from the J.A. Woollam optical constants database [32]. The non-ideal glass-titanium layer was also described using the Bruggeman Effective Medium Approximation model with fractions of glass and titanium 
(set at $1 / 2$ ). Its thickness was established to be $2 \mathrm{~nm}$. The optical constants of glass substrate were established in the separate experiment $[12,13]$.

The Drude-Lorentz model was used to parameterize the complex dielectric function $(\widetilde{\varepsilon})$ of titanium films [31,32]:

$$
\widetilde{\varepsilon}=\varepsilon_{\infty}-\frac{\left(\hbar \omega_{p}\right)^{2}}{E^{2}+(\hbar \Gamma) E}+\sum_{j=1}^{n} \frac{A_{j} E_{j}^{2}}{E_{j}^{2}-E^{2}-i B r_{j} E} .
$$

In Equation (3), $\varepsilon_{\infty}$ is the high-frequency dielectric constant (set as 1), $\hbar \omega_{p}=E_{p}$, and $\hbar \Gamma=E_{\Gamma}$ are Drude parameters (the unscreened plasma frequency and the free-carrier damping, respectively), while $A_{j}, E_{j}$, and $B r_{j}$ are quantities describing the Lorentzian oscillator (the amplitude, energy, and broadening of the $j$-th absorption band, respectively). The model quantities were varied to minimize the reduced mean squared error, $\chi^{2}$, defined as [32]:

$$
\chi^{2}=\frac{1}{N-P} \sum_{j}\left(\frac{\left(\Psi_{j}^{\text {mod }}-\Psi_{j}^{\exp }\right)^{2}}{\sigma_{\Psi}^{2} j}+\frac{\left(\Delta_{j}^{\text {mod }}-\Delta_{j}^{\text {exp }}\right)^{2}}{\sigma_{\Delta j}^{2}}\right) .
$$

In Equation (4), $N$ is the total number of data points, and $P$ is the number of fitted model parameters. The $\Psi_{j}^{\text {exp }}$ and $\Delta_{j}^{\text {exp }}$ are experimental ellipsometric azimuths. The quantities with superscript 'mod' correspond to the calculated $\Psi$ and $\Delta$ azimuths. The sigma is a standard deviation determined for $\Psi\left(\sigma_{\Psi}\right)$ and $\Delta\left(\sigma_{\Delta j}\right)$ quantities. An example of the fit is presented in Figure $3 \mathrm{~b}$. The determined values of $\chi^{2}$ are lower than 1.6 for all the samples examined in this study.

The estimated thickness of titanium film $\left(d_{T i}\right)$ and the rough layer $\left(d_{E M A}\right)$ are summarized in Table 1. Figure $3 \mathrm{c}$ presents the thickness $\left(d_{T i}\right)$ of titanium coatings as a function of the deposition time $\left(t_{T i}\right)$. The estimated $d_{T i}$ values for Ti layers fabricated using the GIMS method fall within the range from 12 to $1470 \mathrm{~nm}$. Linear dependence $d_{T i}\left(t_{T i}\right)=0.66 t_{T i}+5.3$ (see Figure 3) allows to estimate the deposition rate $\left(d_{r}\right)$ of Ti layers which was established to be $40 \mathrm{~nm} / \mathrm{min}$. The $d_{E M A}$ values are in the range from 1 to $8 \mathrm{~nm}$ and coincide well with the $R_{a}$ values determined from the AFM measurements (see Table 1).

Figure $4 \mathrm{a}, \mathrm{b}$ show the real $\left(\varepsilon_{1}\right)$ and imaginary $\left(\varepsilon_{2}\right)$ parts of the complex dielectric function of $\mathrm{Ti}$ coatings, respectively. To make the figure (Figure 4) more clear, the $\widetilde{\varepsilon}$ curves for chosen thicknesses of titanium layers are drawn. The complex dielectric functions for the other produced Ti coatings are located between the curves presented in Figure 4. The differences in $\varepsilon_{1}$ and $\varepsilon_{2}$ of particular films are visible in the NIR-vis spectral range. This effect has two sources: the change of Drude contribution to the complex dielectric function (see Figure $4 c, d$ ) and, more evident, an increase in maximum in $\varepsilon_{1}$ spectra near $1.0 \mathrm{eV}$ with an increase in the Ti layer thickness. The $\widetilde{\varepsilon}$ demonstrates typical features for Ti metallic samples: the Drude term in the IR spectral range and interband transition at 3.0-3.3 eV. In the inset of Figure $4 \mathrm{~d}$ the Lorentzian contribution $\left(\varepsilon_{2 L}\right)$ to the complex dielectric function is presented. The shape of $\varepsilon_{2 L}$ exhibits two maximums. The first absorption feature, located at about $3 \mathrm{eV}$, is associated with the interband transitions of Ti. The second one (at about 1.0-1.5 eV) slightly shifts toward longer wavelengths with an increase in titanium layer thickness and is related to the granular structure of the titanium films. Most probably, these resonance features are related to plasmonic effect associated with a granular structure of Ti films and was observed earlier for pure metals [33] and alloys [34,35]. 
(a)
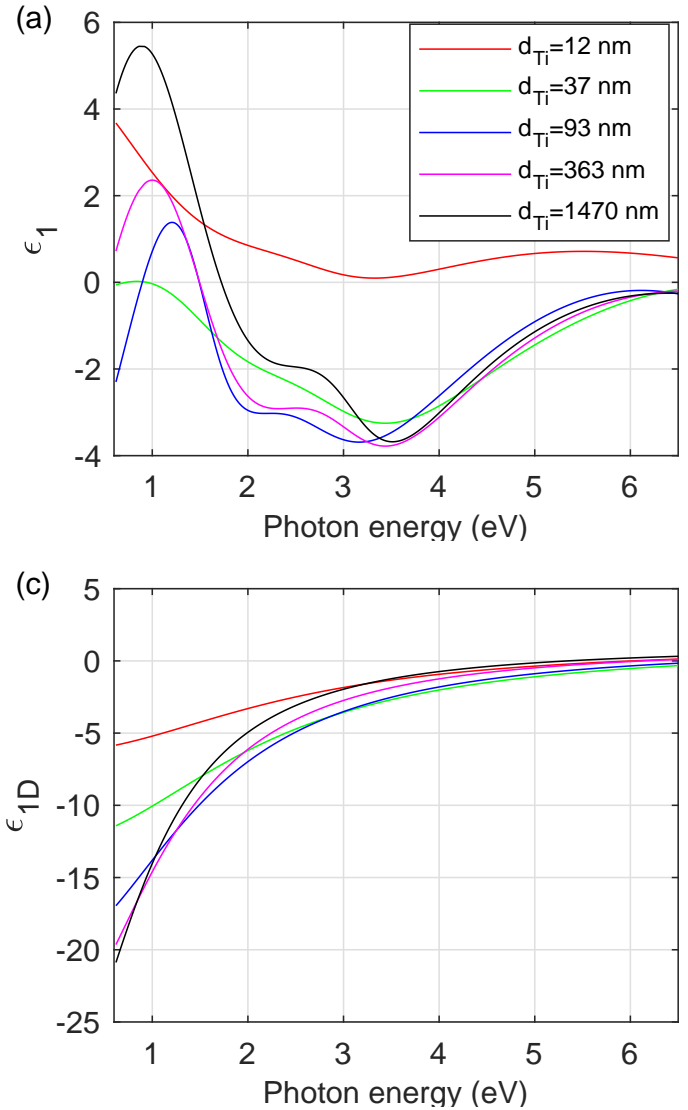

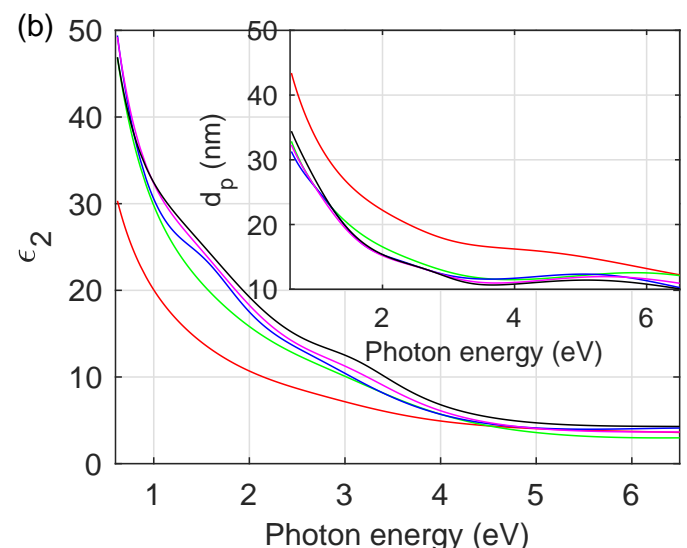

(d)

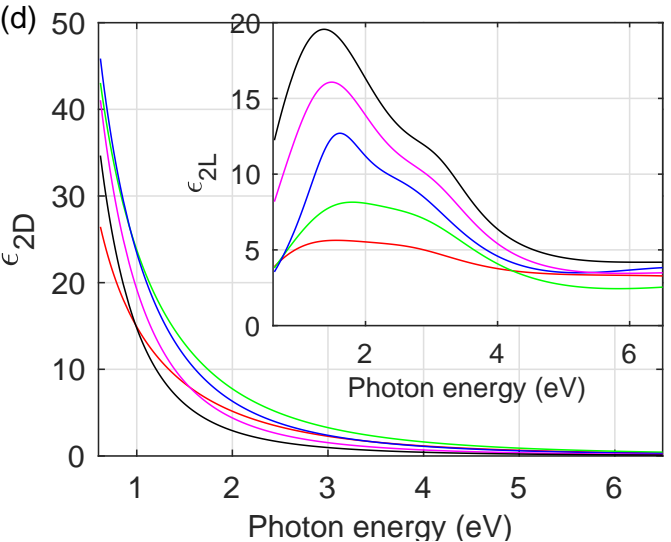

Figure 4. (a) The real $\left(\varepsilon_{1}\right)$ and (b) imaginary $\left(\varepsilon_{2}\right)$ parts of the complex dielectric function $(\widetilde{\varepsilon})$ of the titanium films. Inset: the penetration depth $\left(d_{p}\right)$. (c) The real $\left(\varepsilon_{1 D}\right)$ and $(\mathbf{d})$ imaginary $\left(\varepsilon_{2 D}\right)$ parts of the Drude contribution to $\widetilde{\varepsilon}$. Inset: The separate contributions from the Lorentz terms $\left(\varepsilon_{2 L}\right)$ to the imaginary part of $\widetilde{\mathcal{E}}$. The complex dielectric function of the Ti films of thickness equals $12 \mathrm{~nm}$ and $37 \mathrm{~nm}$ is taken from Reference [13].

To obtain detailed description of absorption features related to the free-electrons, the Drude parameters $\left(\hbar \omega_{p}\right.$ and $\left.\hbar \Gamma\right)$ were determined during the analysis of spectroscopic ellipsometry results. The estimated values of $\hbar \omega_{p}$ and $\hbar \Gamma$ are summarized in Table 2. The Drude parameters $\hbar \omega_{p}$ and $\hbar \Gamma$ are associated with the concentration and scattering frequency of free electrons, respectively. Based on their values, two additional parameters describing the free-carriers can be calculated. The mean relaxation time of conduction electrons is defined as:

$$
\tau=\Gamma^{-1},
$$

while the optical resistivity at $\hbar \omega=0$ (corresponding to the dc-resistivity):

$$
\rho_{o}=\frac{\hbar \Gamma}{\left(\hbar \omega_{p}\right)^{2}}
$$

Table 2. The time of deposition $\left(t_{T i}\right)$, the thickness of titanium film $\left(d_{T i}\right)$ and the EMA layer $\left(d_{E M A}\right)$, the roughness parameter $\left(R_{a}\right)$ and the average size of nanocrystallites $(<D>)$.

\begin{tabular}{cccccc}
\hline Sample & $\boldsymbol{t}_{\boldsymbol{T} i}(\mathbf{s})$ & $\boldsymbol{d}_{\boldsymbol{T} i}(\mathbf{n m})$ & $\boldsymbol{d}_{\boldsymbol{E M A}}(\mathbf{n m})$ & $\boldsymbol{R}_{\boldsymbol{a}}(\mathbf{n m})$ & $\langle\boldsymbol{D}\rangle \mathbf{( n m )}$ \\
\hline $\operatorname{Ti}(12)^{*}$ & 11 & $12.2 \pm 0.1^{a}$ & $2.0 \pm 0.1$ & $2.1 \pm 0.2$ & $-^{c}$ \\
$\operatorname{Ti}(16)^{*}$ & 15 & $15.5 \pm 0.2^{a}$ & $1.7 \pm 0.1$ & $1.8 \pm 0.5$ & $-^{c}$ \\
\hline
\end{tabular}


Table 2. Cont.

\begin{tabular}{cccccc}
\hline Sample & $\boldsymbol{t}_{\boldsymbol{T} i}(\mathbf{s})$ & $\boldsymbol{d}_{\boldsymbol{T i}}(\mathbf{n m})$ & $\boldsymbol{d}_{\boldsymbol{E M A} \boldsymbol{A}}(\mathbf{n m})$ & $\boldsymbol{R}_{\boldsymbol{a}}(\mathbf{n m})$ & $\langle\boldsymbol{D}\rangle \mathbf{( n m})$ \\
\hline $\operatorname{Ti}(21)^{*}$ & 22 & $20.8 \pm 0.3^{a}$ & $1.5 \pm 0.1$ & $1.8 \pm 0.3$ & $-^{c}$ \\
$\operatorname{Ti}(26)^{*}$ & 30 & $26.2 \pm 0.1^{a}$ & $2.1 \pm 0.1$ & $2.3 \pm 0.3$ & $-^{c}$ \\
$\operatorname{Ti}(37)^{*}$ & 45 & $38.6 \pm 0.1^{a}$ & $2.9 \pm 0.1$ & $2.7 \pm 0.2$ & $-^{c}$ \\
$\operatorname{Ti}(49)^{*}$ & 60 & $48.8 \pm 0.2^{a}$ & $1.2 \pm 0.1$ & $3.0 \pm 0.5$ & $-^{c}$ \\
$\operatorname{Ti}(93)$ & 150 & $93 \pm 7^{b}$ & $3.4 \pm 0.6$ & $2.2 \pm 0.2$ & $8 \pm 5$ \\
$\operatorname{Ti}(164)$ & 260 & $164 \pm 10^{b}$ & $4.2 \pm 0.7$ & $2.0 \pm 0.2$ & $10 \pm 4$ \\
$\operatorname{Ti}(363)$ & 530 & $363 \pm 22^{b}$ & $4.8 \pm 0.7$ & $3.8 \pm 0.2$ & $7 \pm 3$ \\
$\operatorname{Ti}(743)$ & 1120 & $743 \pm 11^{b}$ & $5.4 \pm 0.7$ & $5.8 \pm 0.5$ & $7 \pm 3$ \\
$\operatorname{Ti}(1470)$ & 2240 & $1470 \pm 40^{b}$ & $8.0 \pm 1.4$ & $8.3 \pm 2.7$ & $7 \pm 3$
\end{tabular}

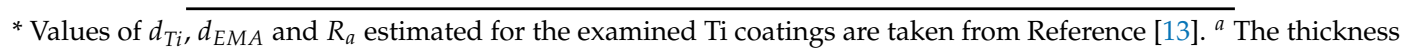
of Ti coating determined using spectroscopic ellipsometry. ${ }^{b}$ The thickness of Ti coating determined using confocal optical microscope. ${ }^{c}$ Diffractograms were not recorded.

The determined values of $\hbar \omega_{p}, \hbar \Gamma, \tau$ and $\rho_{o}$ for the Ti coatings are summarized in Table 2. In order to observe more easily the trends in changes of $\hbar \omega_{p}, \hbar \Gamma, \tau$ and $\rho_{o}$ parameters, their values have been plotted (Figure 5) as a function of the thickness of titanium coating. Figure 5a,b present the plasma energy $\left(\hbar \omega_{p}=E_{p}\right)$, the free carrier dumping $\left(\hbar \Gamma=E_{\Gamma}\right)$, as well as the mean relaxation time of conduction electrons $(\tau)$, respectively. The $\hbar \omega_{p}$ value increases from $6.4 \mathrm{eV}$ to $8.7 \mathrm{eV}$ with the increase in the thickness of the titanium coating from 12 to $93 \mathrm{~nm}$ and then decreases from $8.7 \mathrm{eV}$ to $5.7 \mathrm{eV}$ for larger values of $d_{T i}$. The mean relaxation time of conduction electrons $(\tau)$ increases from $0.27 \mathrm{eV}$ (for the Ti(12) sample) to $0.60 \mathrm{eV}$ (for the Ti(1470) sample). It should be noted that, for most metallic coatings deposited at established growth conditions (only time of deposition is varied, thus varying the thickness of a layer), the influence of the thickness of a layer on the value of $\tau$ (and $\Gamma$ ) shows the tendency presented in Figure $5 \mathrm{a}, \mathrm{b}$; however, the determined $\hbar \omega_{p}\left(d_{T i}\right)$ relation exhibits the pronounced maximum $\left(\hbar \omega_{p}(93 \mathrm{~nm})=8.7 \mathrm{eV}\right)$. Both $\hbar \omega_{p}$, as well as $\tau$, directly affect the $\rho_{o}$ value (Equations (5) and (6)). The $\rho_{o}$ for the thinnest Ti coating was established to be $422 \mu \Omega \mathrm{cm}$; for the thickest, it was $250 \mu \Omega \mathrm{cm}$. However, the minimum value of $\rho_{o}=202 \mathrm{eV}$ was obtained for the sample with $d_{T i}=93$ $\mathrm{nm}$, that is, for the coating with the highest value of $\hbar \omega_{p}$.

To explain the unexpected tendency in $E p\left(d_{T i}\right)$ and $\rho_{o}\left(d_{T i}\right)$ (the existence of extrema) the 4PP measurements were performed for all the samples prepared. A sheet resistance $(R)$ values are in the range from $434 \Omega$ for the Ti(12) sample to about $2 \Omega$ for the Ti(1470) specimen and are summarized in Table 2. The sheet resistance of the Ti coatings demonstrates behavior typical for metals; $R$ value decreases with $d_{T i}$ increasing, which is presented in Figure $5 \mathrm{c}$. The dc-resistivity of the produced titanium coatings $\left(\rho_{d c}=R d_{T i}\right)$ decreases from $529 \mu \Omega \mathrm{cm}$ to $162 \mu \Omega \mathrm{cm}$ with the increase in the thickness of the Ti layer from 12 to $1470 \mathrm{~nm}$ (see Table 2). For semitransparent titanium coatings $\left(d_{T i} \leq 49 \mathrm{~nm}\right)$ [13] the $\rho_{d c}$ values are higher than adequate $\rho_{o}$ ones. The discrepancies are lower than $5 \%$ for the titanium films with $d_{T i}=93 \mathrm{~nm}$ and are about $20 \%$ for the Ti layer with $d_{T i}=12 \mathrm{~nm}$. This agreement is satisfactory, considering a completely different experimental methods used to determine $\rho_{o}$ and $\rho_{d c}$ quantities. For the thicker Ti layers $\left(d_{T i} \geq 93 \mathrm{~nm}\right)$ the value of $\rho_{d c}$ still decreases with increase in the $d_{T i}$. It should be noted that apart from the quite significant percentage difference between $\rho_{o}$ and $\rho_{d c}$ (up to $40 \%$ ), the behavior of $\rho_{d c}$ is opposite to the behavior of $\rho_{o}$ (i.e., the decrease in $\rho_{d c}$ with the increase in $d_{T i}$ ). 

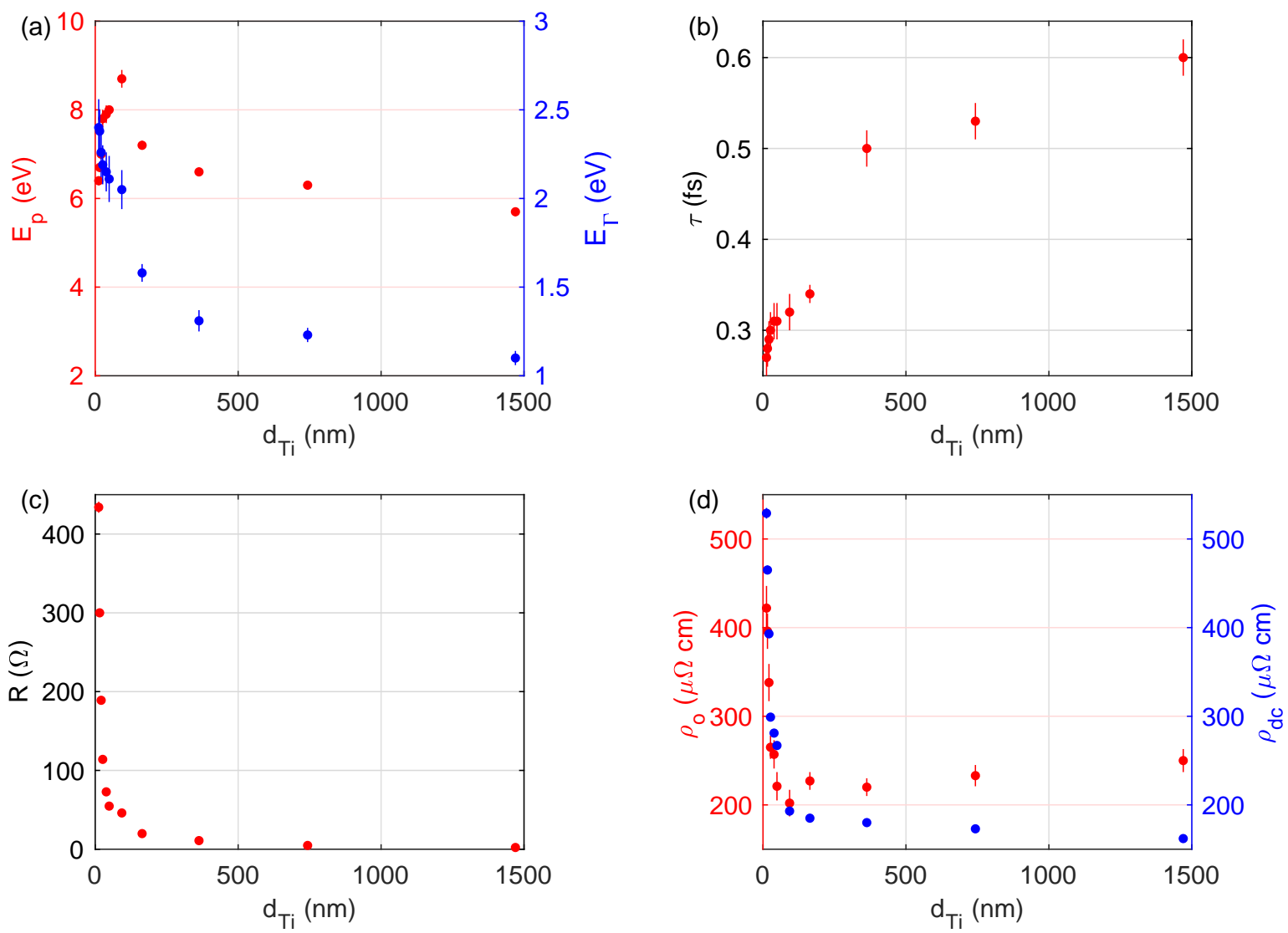

Figure 5. (a) The plasma energy $\left(E_{p}=\hbar \omega_{p}\right)$ and the free-carrier dumping $\left(E_{\Gamma}=\hbar \Gamma\right)$, (b) the mean relaxation time of conduction electrons $(\tau),(\mathbf{c})$ the sheet resistance $(R)$, and $(\mathbf{d})$ the optical $\left(\rho_{o}\right)$ and dc-resistivity $\left(\rho_{d c}\right)$ determined for the examined Ti coatings plotted as a function of the thickness of the Ti film.

According to the hypothesis based on the Matthiesen's rule, the electrical resistivity $\left(\rho_{d c}\right)$ of a non-ideal metal film can be expressed as:

$$
\rho_{d c}=\rho_{d c 0}+\rho_{g b}+\rho_{s s}+\rho_{s r}
$$

where $\rho_{d c 0}$ is the residual resistivity, and $\rho_{g b}, \rho_{s s}$ and $\rho_{s r}$ are contribution from grain-boundary, surface and surface roughness scattering, respectively. For thinner coatings the main factors influence the resistivity are related to the film-substrate and film-ambient interfaces, as well as the surface roughness. For thicker layers the thickness of a film (and in the presented study limited surface roughness) is less evident. The coatings with larger grains (thicker layers) contain relatively a few grain boundaries and this effect plays a major role in increasing of the mean relaxation time of conduction electrons, whereas the plasma energy decreasing with an increase in the layer thickness is associated with the density of the material. For thicker films the subsurface structure is less packed (see Figure 1). The presented clarification explains correctly the typical behavior of $\rho_{d c}$ for $12 \mathrm{~nm} \leq d_{T i} \leq 1470 \mathrm{~nm}$ and $\rho_{o}$ for $d_{T i} \leq 93 \mathrm{~nm}$ (see Figure $5 \mathrm{~d}$ ); however, the behavior of $\rho_{o}$ for the thicker titanium coatings $\left(d_{T i}>93 \mathrm{~nm}\right)$ is still unexplained. The discrepancies between $\rho_{o}$ and $\rho_{d c}$ values for thicker titanium coatings can be explained as an effect of limited light penetration. Figure $4 \mathrm{~b}$ (the inset) shows the penetration depth [32]:

$$
d_{p}=\frac{\lambda}{4 \pi k}
$$

of light as a function of photon energy. In Equation (8), $\lambda$ is a wavelength, and $k$ is the extinction coefficient $(k=\operatorname{Im}(\sqrt{\widetilde{\varepsilon}})$ ). This quantity informs about the depth from the surface where the light 
intensity decreases $e$-times. The maximum $d_{p}$ value falls in the NIR spectral range and does not exceed $45 \mathrm{~nm}$ for the thinnest layer and it is lower than $35 \mathrm{~nm}$ for the thicker Ti coatings. In the visible and ultraviolet spectral range the $d_{p}$ value is lower than $15 \mathrm{~nm}$ (20 nm for the thinner Ti film). However, if the layer thickness is higher than the $d_{p}$ value, determining its thickness is still possible. Spectroscopic ellipsometry has a very high precision up to thickness $\sim 5 \mathrm{~d}_{p}[31,36]$. Therefore, in an optical model for thinner samples $\left(d_{T i} \leq 49 \mathrm{~nm}\right)$, the substrate (Bk7 glass) was considered. The thicknesses of the other titanium films examined are larger (except the Ti(93) sample) than $\sim 5 \mathrm{~d}_{p}$ (see Table 1) and, consequently, during the SE measurement, the optical response to incident electromagnetic radiation was recorded only from a top part of the Ti coating. Therefore, the determined complex dielectric function and parameters describing opto-electronic properties of layers $\left(\hbar \omega_{p}, \hbar \Gamma, \tau\right.$ and $\left.\rho_{o}\right)$ do not represent whole coating; however, they mainly contain information about their subsurface structure. Due to the relatively high thickness of the titanium coatings investigated $\left(d_{T i}>93 \mathrm{~nm}\right)$ and the limited penetration depth of the electromagnetic radiation into the layer, the opto-electronic properties of Ti films should be discussed taking this fact into account. These facts explain the behavior of the plasma energy $\left(\hbar \omega_{p}\right)$ and the optical resistivity $\left(\rho_{o}\right)$ and thus discrepancies between the optical and dc-resistivities for the thicker titanium films $\left(d_{T i}>93 \mathrm{~nm}\right)$.

\section{Conclusions}

The optical, electrical, and microstructural properties of titanium coatings of thickness from 12 to $1470 \mathrm{~nm}$ produced using the GIMS technique were examined by means of atomic force microscopy, X-ray diffraction, spectroscopic ellipsometry, confocal optical microscopy, and a four-point probe techniques. The prepared layers exhibit granular structure, which affects their optical properties. The Ti films show the privileged direction of growth (002). The average size of nanocrystallites was established to be 7-10 nm. The complex dielectric function of Ti films is typical for conducting materials: the free-carrier absorption, interband transitions, and additional absorption related to the granular structure of a coating. The free-carrier dumping $(\hbar \Gamma)$ and the mean relaxation time of conduction electrons $(\tau)$ exhibit a typical behavior for metallic coatings; however, the plasma energy $\left(\hbar \omega_{p}\right)$ and the optical resistivity $\left(\rho_{o}\right)$ show extreme behavior, which can be ascribed to the granular structure of the top part of a Ti coating. The differences between optical $\left(\rho_{o}\right)$ and dc-resistivities $\left(\rho_{d c}\right)$ can be explained by the limited penetration depth of light, much less in comparison with the thickest films.

Funding: Financial support to instrumentation has been obtained from the European Regional Development Fund (Stage 2 of the Regional Centre of Innovativeness) and from the Polish Ministry of Science and Higher Education.

Acknowledgments: Special thanks are due to M.K. Naparty, A. Wronkowski (both Institute of Mathematics and Physics, UTP University of Science and Technology, Bydgoszcz, Poland) and M. Kobierski (Department of Soil Science and Soil Protection, UTP University of Science and Technology, Bydgoszcz, Poland) for AFM, 4PP and XRD measurements. The author would also like to thank Aleksandra A. Wronkowska for fruitful discussion.

Conflicts of Interest: The authors declare no conflict of interest.

\section{References}

1. Thornton, J.A. Influence of apparatus geometry and deposition conditions on the structure and topography of thick sputtered coatings. J. Vac. Sci. Technol. 1974, 11, 666-670. [CrossRef]

2. Chawla, V.; Jayaganthan, R.; Chawla, A.K.; Chandra, R. Morphological study of magnetron sputtered Ti thin films on silicon substrate. Mater. Chem. Phys. 2008, 111, 414-418. [CrossRef]

3. Chawla, V.; Jayaganthan, R.; Chawla, A.K.; Chandra, R. Microstructural characterizations of magnetron sputtered Ti films on glass substrate. J. Mater. Process. Technol. 2009, 209, 3444-3451. [CrossRef]

4. Zhang, L.; Shia, L.Q.; He, Z.J.; Zhang, B.; Lu, Y.F.; Liu, A.; Wang, B.Y. Deposition of dense and smooth Ti films using ECR plasma-assisted magnetron sputtering, Surf. Coat. Technol. 2009, 203, 3356-3360. [CrossRef]

5. Jin, Y.; Wu, W.; Li, L.; Chen, J.; Zhang, J.; Zuo, Y.; Fu, J. Effect of sputtering power on surface topography of dc magnetron sputtered Ti thin films observed by AFM. Appl. Surf. Sci. 2009, 255, 4673--4679. [CrossRef] 
6. Jung, M.J.; Nam, K.H.; Shaginyan, L.R.; Han, J.G. Deposition of Ti thin film using the magnetron sputtering method. Thin Solid Films 2003, 435, 145-149. [CrossRef]

7. Martin, N.; Baretti, D.; Rousselot, C.; Rauch, J.Y. The effect of bias power on some properties of titanium and titanium oxide films prepared by r.f. magnetron sputtering. Surf. Coat. Technol. 1998, 107, 172-182. [CrossRef]

8. Šícha, J.; Musil, J.; Meissner, M.; Čerstvý, R. Nanostructure of photocatalytic TiO2 films sputtered at temperatures below $200^{\circ} \mathrm{C}$. Appl. Surf. Sci. 2008, 254, 3793-3800. [CrossRef]

9. Zdunek, K.; Nowakowska-Langier, K.; Dora, J.; Chodun, R. Gas injection as a tool for plasma process control during coating deposition. Surf. Coat. Technol. 2013, 228, S367-S373. [CrossRef]

10. Zdunek, K.; Nowakowska-Langier, K.; Chodun, R.; Okrasa, S.; Rabiński, M.; Dora, J.; Domanowski, P.; Halarewicz, J. Impulse Plasma In Surface Engineering-a review. J. Phys. Conf. Ser. 2014, 564, 012007. [CrossRef]

11. Zdunek, K.; Skowronski, L.; Chodun, R.; Nowakowska-Langier, K.; Grabowski, A.; Wachowiak, W.; Okrasa, S.; Wachowiak, A.A.; Strauss, O.; et al. Nowel GIMS technique for deposition of colored $\mathrm{Ti} / \mathrm{TiO}_{2}$ coatings in industrial scale. MSP 2016, 34, 137-141.

12. Skowroński, Ł.; Trzcinski, M.; Antończak, A.J.; Domanowski, P.; Kustra, M.; Wachowiak, W.; Naparty, M.K.; Hiller, T.; Bukaluk, A.; Wronkowska, A.A. Characterisation of coloured $\mathrm{TiO}_{x} / \mathrm{Ti} /$ glass systems. Appl. Surf. Sci. 2014, 322, 209-214.

13. Skowronski, L.; Wachowiak, A.A.; Grabowski, A. Characterization of optical and microstructural properties of semitransparent $\mathrm{TiO}_{2} / \mathrm{Ti} /$ glass interference decorative coatings. Appl. Surf. Sci. 2016, 388, 731-740. [CrossRef]

14. Skowronski, L.; Wachowiak, A.A.; Wachowiak, W. Optical and microstructural properties of decorative $\mathrm{Al} / \mathrm{Ti} / \mathrm{TiO}_{2}$ interference coatings. Appl. Surf. Sci. 2017, 421, 794-801. [CrossRef]

15. Skowronski, L.; Wachowiak, A.A.; Zdunek, K.; Trzcinski, M.; Naparty, M.K. TiO2-based decorative coatings deposited on the AISI 316L stainless steel and glass using an industrial scale magnetron. Thin Solid Films 2017, 627, 1-8. [CrossRef]

16. Chodun, R.; Skowronski, L.; Okrasa, S.; Wicher, B.; Nowakowska-Langier, K.; Zdunek, K. Optical TiO 2 layers deposited on polymer substrates by the Gas Injection Magnetron Sputtering technique. Appl. Surf. Sci. 2019, 466, 12-18. [CrossRef]

17. Skowronski, L.; Zdunek, K.; Nowakowska-Langier, K.; Chodun, R.; Trzcinski, M.; Kobierski, M.; Kustra, M.K.; Wachowiak, A.A.; Wachowiak, W.; Hiller, T.; et al. Characterization of microstructural, mechanical and optical properties of $\mathrm{TiO}_{2}$ layers deposited by GIMS and PMS methods. Surf. Coat. Technol. 2015, 282, 16-23. [CrossRef]

18. Henderson, P.S.; Kelly, P.J.; Arnell, R.D.; Bäcker, H.; Bradley, J.W. Investigation into the properties of titanium based films deposited using pulsed magnetron sputtering. Surf. Coat. Technol. 2003, 174-175, 779-783. [CrossRef]

19. Hofmann, K.; Spangenberg, B.; Luysberg, M.; Kurz, H. Properties of evaporated titanium thin films and their possible application in single electron devices. Thin Solid Films 2003, 436, 168-174. [CrossRef]

20. Jeyachandran, Y.L.; Karunagaran, B.; Narayandass, S.K.; Mangalaraj, D. The effect of thickness on the properties of titanium films deposited by dc magnetron sputtering. Mater. Sci. Eng. A 2007, 458, 361-365. [CrossRef]

21. Jeyachandran, Y.L.; Karunagaran, B.; Narayandass, S.K.; Mangalaraj, D.; Jenkins, T.E.; Martin, P.J. Properties of titanium thin films deposited by dc magnetron sputtering. Mater. Sci. Eng. A 2006, 431, 277-284. [CrossRef]

22. Rakić, A.D.; Djurišić, A.B.; Elazar, J.M.; Majewski, M.L. Optical properties of metallic films for vertical-cavity optoelectronic devices. Appl. Opt. 1998, 37, 5271-5283. [CrossRef] [PubMed]

23. Mahmoud, S. Structure and optical properties of thin titanium films deposited on different substrates. J. Mater. Sci. 1987, 22, 3693-3697. [CrossRef]

24. Johnson, P.B.; Christy, R.W. Optical constants of transition metals: Ti, V, Cr, Mn, Fe, Co, Ni, and Pd. Phys. Rev. B 1974, 9, 5056-5070. [CrossRef]

25. Domanowski, P.; Wawrzak, A. Automation of thin film deposition process based on magnetron sputtering. J. Mach. Eng. 2012, 2, 111-119.

26. Posadowski, W.M.; Wiatrowski, A.; Dora, J.; Radzimski, Z.J. Magnetron sputtering process control by medium-frequency power supply parameter. Thin Solid Films 2008, 516, 4478-4482. [CrossRef] 
27. Wicher, B.; Chodun, R.; Nowakowska-Langier, K.; Trzcinski, M.; Skowronski, L.; Okrasa, S.; Minikayev, R.; Naparty, M.K.; Zdunek, K. Chemical and structural characterization of tungsten nitride $\left(\mathrm{WN}_{x}\right)$ thin films synthesized via Gas Injection Magnetron Sputtering technique. Vacuum 2019, 165, 266-273. [CrossRef]

28. Strzelecki, G.W.; Nowakowska-Langier, K.; Chodun, R.; Okrasa, S.; Wicher, B.; Zdunek, K. Influence of modulation frequency on the synthesis of thin films in pulsed magnetron sputtering processes. MSP 2018, 36, 697-703. [CrossRef]

29. Wicher, B.; Chodun, R.; Kwiatkowski, R.; Trzcinski, M.; Nowakowska-Langier, K.; Lachowski, A.; Minikayev, R.; Rudnicki, J.; Naparty, M.K.; Zdunek, K. Plasmochemical investigations of DLC/WC $x$ nanocomposite coatings synthesized by gas injection magnetron sputtering technique. Diam. Relat. Mater. 2019, 96, 1-10. [CrossRef]

30. Yakubowski, D.I.; Arsenin, A.V.; Stebunov, Y.V.; Fedyanin, D.Y.; Volkov, V.S.; Optical constants and structural properties of thin gold films. Opt. Express 2017, 25, 25574-25587.

31. Fujiwara, H. Spectroscopic Ellipsometry. Principles and Applications: John Wiley \& Sons Ltd: Chichester, UK, 2009.

32. Woollam, J.A. Guide to Using WVASE32®, Wextech Systems Inc.: New York, NY, USA, 2010.

33. Rerek, T.; Skowronski, L.; Szczesny, R.; Naparty, M.K.; Derkowska-Zielinska, B. The effect of the deposition rate on microstructural and opto-electronic properties of $\beta$-Sn layers. Thin Solid Films 2019, 670, 86-92. [CrossRef]

34. Rerek, T.; Skowronski, L.; Kobierski, M.; Naparty, M.K.; Derkowska-Zielinska, B. Microstructure and opto-electronic properties of Sn-rich Au-Sn diffusive solders. Appl. Surf. Sci. 2018, 451, 32-39. [CrossRef]

35. Wronkowska, A.A.; Czerniak, G.; Wronkowski, A.; Skowronski, L. Optical and microstructural characterisation of Au-Sn and Cu-Sn diffusive layers. Appl. Surf. Sci. 2013, 281, 30-37. [CrossRef]

36. Wakagi, M.; Fujiwara, H.; Collins, R.W. Real time spectroscopic ellipsometry for characterization of the crystallization of amorphous silicon by thermal annealing. Thin Solid Films 1998, 313-314, 464-468. [CrossRef]

(C) 2020 by the authors. Licensee MDPI, Basel, Switzerland. This article is an open access article distributed under the terms and conditions of the Creative Commons Attribution (CC BY) license (http://creativecommons.org/licenses/by/4.0/). 